MATHEMATICS OF COMPUTATION

Volume 68, Number 228, Pages 1631-1647

S $0025-5718(99) 01130-8$

Article electronically published on May 17, 1999

\title{
AN ATLAS OF REGULAR THIN GEOMETRIES FOR SMALL GROUPS
}

\author{
DIMITRI LEEMANS
}

\begin{abstract}
For some small groups, we give, up to isomorphism, an exhaustive list of all residually connected thin geometries on which these groups act regularly. We then show the utility of such an atlas by proving several results about smallest groups acting on a given diagram. The results have been obtained using a series of MAGMA programs.
\end{abstract}

\section{INTRODUCTION}

Several years ago, a systematic investigation of group-geometry pairs satisfying a set of axioms started in Brussels (see [7, 9, 8, 18, 19, 20, 22, 23, 27]). This experimental work gave rise to several theoretical results (see $[6,5,10,12,14,15$, $25])$. Some of these publications are the results of a more ambitious projectthat is, to classify all geometries satisfying a set of axioms for an infinite family of groups. Families that are currently under investigation are the symmetric groups and the Suzuki simple groups. The dihedral groups have been studied in [24]. While working on the classification for the Suzuki groups, we found lots of thin geometries arising. We already noticed during the experimental work cited above that if thin geometries appear for a given group, there are generally lots of them. This leads us to classify all thin and residually connected geometries on which a group $G$ acts flag-transitively, for several small groups.

This paper is organized as follows. In Section 2, we give the basic definitions and notation. In Section 3, we give, for $\operatorname{Alt}(5), \operatorname{PSL}(2,7)$, Alt $(6), \operatorname{PSL}(2,8)$, $P S L(2,11)$, and $M_{11}$, an exhaustive list of all their thin, residually connected, and flag-transitive geometries. The group $S z(8)$ has already been studied in [26]. In Section 4, we do the same for some non-simple groups, namely $\operatorname{Alt}(4), \operatorname{Alt}(4) \times 2$, $\operatorname{Sym}(4), \operatorname{Alt}(5) \times 2, \operatorname{Sym}(5), \operatorname{Sym}(5) \times 2, P G L(2,7)$, and $M_{10}$. In Section 5, we give some theoretical results that were conjectured by looking at the lists of geometries obtained in the previous sections. This section shows the interest of building such an atlas. In Section 6, we show the connection between some of our thin geometries and reflexive maps described in [17]. Finally in Section 7, we talk about Petrie polygons, and we give examples of polyhedra for which the Petrial is not a polyhedron anymore.

Received by the editor February 10, 1998.

1991 Mathematics Subject Classification. Primary 51E24, 52B10, $20 B 99$.

Key words and phrases. Incidence geometry, group theory, regular maps, polytopes.

This research was accomplished during a stay at the University of Sydney. We gratefully acknowledge support from the Fonds National de la Recherche Scientifique de Belgique and The University of Sydney. 
Acknowledgments. We would like to thank Francis Buekenhout for many interesting discussions and for useful comments, as well as suggesting the choice of groups, while building this atlas. We also would like to thank Michel Dehon and Harold Goltschalk for their comments on a preliminary version of this paper. Finally, we thank John Cannon for his invitation to come to the University of Sydney, where part of this work was done.

\section{Definitions and notation}

The basic concepts about geometries constructed from a group and some of its subgroups are due to Tits [29] (see also [4], chapter 3).

Let $G$ be a group together with a finite family of subgroups $\left(G_{i}\right)_{i \in I}$. We define the pre-geometry $\Gamma=\Gamma\left(G,\left(G_{i}\right)_{i \in I}\right)$ as follows. The set $X$ of elements of $\Gamma$ consists of all cosets $g G_{i}, g \in G, i \in I$. We define an incidence relation $*$ on $X$ by

$$
g_{1} G_{i} * g_{2} G_{j} \quad \text { iff } \quad g_{1} G_{i} \cap g_{2} G_{j} \text { is non-empty in } G \text {. }
$$

The type function $t$ on $\Gamma$ is defined by $t\left(g G_{i}\right)=i$. The type of a subset $Y$ of $X$ is the set $t(Y)$; its rank is the cardinality of $t(Y)$ and we call $|I|$ the rank of $\Gamma$. The subgroups $\left(G_{i}\right)_{i \in I}$ are called the maximal parabolic subgroups. The Borel subgroup of the pre-geometry is the $\operatorname{subgroup} B=\bigcap_{i \in I} G_{i}$. A flag is a set of pairwise incident elements of $X$, and a chamber of $\Gamma$ is a flag of type $I$. An element of type $i$ is also called an i-element.

The group $G$ acts on $\Gamma$ as an automorphism group, by left translation, preserving the type of each element.

This action involves a kernel $K$ which is the largest normal subgroup of $G$ contained in every $G_{i}, i \in I$. If the kernel is the identity, we say that $G$ acts faithfully on $\Gamma$. As in [18], we call $\Gamma$ a geometry provided that every flag of $\Gamma$ is contained in some chamber, and we call $\Gamma$ flag-transitive (FT) provided that $G$ acts transitively on all chambers of $\Gamma$, hence also on all flags of any type $J$, where $J$ is a subset of $I$.

If $G$ acts faithfully and flag-transitively on $\Gamma$, we say that $G$ acts regularly on $\Gamma$. Assuming that $\Gamma$ is a flag-transitive geometry and that $F$ is a flag of $\Gamma$, the residue of $F$ is the pre-geometry

$$
\Gamma_{F}=\Gamma\left(\bigcap_{j \in t(F)} G_{j},\left(G_{i} \cap\left(\bigcap_{j \in t(F)} G_{j}\right)\right)_{i \in I \backslash t(F)}\right),
$$

and we readily see that $\Gamma_{F}$ is a flag-transitive geometry.

We call $\Gamma$ firm $(\mathrm{F})$ (resp. thick, thin) provided that every flag of rank $|I|-1$ is contained in at least two (resp. three, exactly two) chambers. We call $\Gamma$ residually connected (RC) provided that the incidence graph of each residue of rank $\geq 2$ is a connected graph. If $\Gamma$ is a geometry of rank 2 with $I=\{0,1\}$ such that each of its 0 -elements is incident with each of its 1-elements, then we call $\Gamma$ a generalized digon.

Following [2] and [3], the diagram of a firm, RC, FT geometry $\Gamma$ is a graph together with additional structure, whose vertices are the elements of $I$, which is further described as follows. To each vertex $i \in I$, we attach the order $s_{i}$ which is $\left|\Gamma_{F}\right|-1$, where $F$ is any flag of type $I \backslash\{i\}$, the number $n_{i}$ of varieties of type $i$, which is the index of $G_{i}$ in $G$, and the subgroup $G_{i}$. Elements $i, j$ of $I$ are not joined by an edge of the diagram provided that a residue $\Gamma_{F}$ of type $\{i, j\}$ is a generalized digon. Otherwise, $i$ and $j$ are joined by an edge endowed with three positive integers 
$d_{i j}, g_{i j}, d_{j i}$ where $g_{i j}$ (the gonality) is equal to half the girth of the incidence graph of a residue $\Gamma_{F}$ of type $\{i, j\}$ and $d_{i j}$ (resp. $d_{j i}$ ), the $i$-diameter (resp. $j$-diameter) is the greatest distance from some fixed $i$-element (resp. $j$-element) to any other element in the incidence graph of $\Gamma_{F}$.

On a picture of the diagram, this structure will often be depicted as follows:

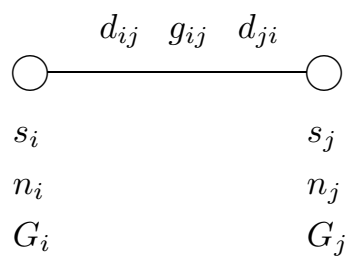

If $g_{i j}=d_{i j}=d_{j i}=n$, then $\Gamma_{F}$ is called a generalized $n$-gon, and on a picture, we do not write $d_{i j}$ and $d_{j i}$.

The ordered pairs $(\Gamma, G)$ and $\left(\Gamma^{\prime}, G\right)$ are isomorphic (resp. conjugate) if there exists an automorphism (resp. inner automorphism) of $G$ mapping $\Gamma$ onto $\Gamma^{\prime}$. The group $\operatorname{Cor}(\Gamma, G)$ (resp. $\operatorname{Aut}(\Gamma, G)$ ) is the group of automorphisms (resp. typepreserving automorphisms) of the pair $(\Gamma, G)$.

As to notation for groups, we follow the conventions of the Atlas [16] up to slight variations. The symbol ":" stands for split extensions, the "hat" symbol "." stands for non-split extensions and the symbol $\times$ stands for direct products. Sometimes we write $A_{5}$ (resp. $S_{5}$ ) instead of $\operatorname{Alt}(5)$ (resp. $\operatorname{Sym}(5)$ ).

The following theorem, due to J. Tits, shows that if a geometry $\Gamma\left(G ;\left(G_{i}\right)_{i \in I}\right)$ has a non-trivial kernel $K$, then this geometry already appeared in $G / K$.

Theorem 1 ([29]). Let $\Gamma\left(G ;\left(G_{i}\right)_{i \in I}\right)$ be a geometry. If $K$ is the kernel of the action of $G$ on $\Gamma$, then $\Gamma\left(G ;\left(G_{i}\right)_{i \in I}\right) \cong \Gamma\left(G / K ;\left(G_{i} / K\right)_{i \in I}\right)$.

This is the reason why we just construct geometries on which the group acts faithfully.

In this Atlas, we look only at geometries $\Gamma$ that are thin and residually connected, and on which the group $G$ acts flag-transitively and faithfully (and hence regularly). The following lemma shows that we then may assume the Borel subgroup of our geometries to be the identity.

Lemma 1. Let $\Gamma\left(G ;\left(G_{i}\right)_{i \in I}\right)$ be a thin geometry on which the group $G$ acts faithfully. Then $B=\bigcap_{i \in I} G_{i}=1$.

Proof. Since $\Gamma$ is thin, the groups $G_{J}=\bigcap_{j \in J} G_{j}$ where $J$ is any subset of $I$ of cardinality $|I|-1$, contain $B$ as a subgroup of index 2 . Hence $B$ is a normal subgroup of all these groups. Now, the subgroups $G_{J}$ generate $G$, and thus $B$ must also be a normal subgroup of $G$. This means $B$ is a kernel. Then in order to have a faithful action of $G$ on $\Gamma$, we must have $B=1$.

\section{Simple GROUPS}

The following tables are read as follows. The first column gives a number to each geometry, and the number in parenthesis is the number of conjugacy classes of geometries that are fused under the action of the automorphism group of the group. The columns labelled $i-j$ give the parameters to put on the edge $i-j$ of the diagram. The column $\operatorname{Cor}(\Gamma, G)$ gives the correlation group. When the diagram 
tells us that $\operatorname{Cor}(\Gamma, G)=\operatorname{Aut}(\Gamma, G)$, we write nothing in this column. We do not mention the automorphism groups, because $\operatorname{Aut}(\Gamma, G)=G$ for every thin geometry. The column $G_{0}, G_{1}, G_{2}, \ldots, G_{n-1}$ gives the maximal parabolic subgroups $G_{0}, G_{1}$, $G_{2}, \ldots, G_{n-1}$.

3.1. Alt (5).

\begin{tabular}{||c|c|c|c|c||}
\hline Nr. & $1-2$ & $1-3$ & $2-3$ & $\operatorname{Cor}(\Gamma, G)$ \\
\hline $1(2)$ & 2 & 3 & 5 & \\
\hline $2(1)$ & 2 & 5 & 5 & $\operatorname{Sym}(5)$ \\
\hline $3(2)$ & 3 & 3 & 5 & $\operatorname{Alt}(5) \times 2$ \\
\hline $4(2)$ & 3 & 5 & 5 & $\operatorname{Alt}(5) \times 2$ \\
\hline
\end{tabular}

3.2. $\operatorname{PSL}(\mathbf{2}, \mathbf{7})$.

\begin{tabular}{||c|c|c|c|c||}
\hline Nr. & $1-2$ & $1-3$ & $2-3$ & $\operatorname{Cor}(\Gamma, G)$ \\
\hline $1(1)$ & 3 & 3 & 4 & $\operatorname{PGL}(2,7)$ \\
\hline $2(1)$ & 3 & 4 & 4 & $\operatorname{PGL}(2,7)$ \\
\hline $3(1)$ & 4 & 4 & 4 & $\operatorname{PSL}(2,7): \operatorname{Sym}(3)$ \\
\hline
\end{tabular}

\begin{tabular}{||c|c|c|c|c|c|c|c|c||}
\hline Nr. & $1-2$ & $1-3$ & $1-4$ & $2-3$ & $2-4$ & $3-4$ & $\operatorname{Cor}(\Gamma, G)$ & $G_{0}, G_{1}, G_{2}, G_{3}$ \\
\hline $4(1)$ & 3 & 4 & 2 & 2 & 3 & 3 & $\operatorname{PGL}(2,7)$ & $S_{4}, S_{4}, S_{4}, S_{4}$ \\
\hline $5(1)$ & 3 & 2 & 4 & 4 & 2 & 3 & $\operatorname{PGL}(2,7) \times 2$ & $S_{4}, S_{4}, S_{4}, S_{4}$ \\
\hline
\end{tabular}

3.3. $\operatorname{Alt}(6)=\operatorname{PSL}(\mathbf{2}, 9)$.

\begin{tabular}{||c|c|c|c|c||}
\hline Nr. & $1-2$ & $1-3$ & $2-3$ & $\operatorname{Cor}(\Gamma, G)$ \\
\hline $1(2)$ & 3 & 3 & 4 & $\operatorname{PGL}(2,9)$ \\
\hline $2(2)$ & 3 & 3 & 5 & $\operatorname{PGL}(2,9)$ \\
\hline $3-4(4)$ & 3 & 4 & 5 & $\operatorname{Alt}(6)$ \\
\hline $5(2)$ & 3 & 5 & 5 & $\operatorname{Sym}(6)$ \\
\hline $6(2)$ & 4 & 4 & 5 & $\operatorname{PGL}(2,9)$ \\
\hline $7(2)$ & 4 & 5 & 5 & $\operatorname{PGL}(2,9)$ \\
\hline
\end{tabular}

\section{4. $\operatorname{PSL}(2,8)$.}

\begin{tabular}{||c|c|c|c|c||}
\hline Nr. & $1-2$ & $1-3$ & $2-3$ & $\operatorname{Cor}(\Gamma, G)$ \\
\hline $1(3)$ & 2 & 3 & 7 & \\
\hline $2(3)$ & 2 & 3 & 9 & \\
\hline $3(3)$ & 2 & 7 & 7 & $\operatorname{PSL}(2,8)$ \\
\hline $4-6(3)$ & 2 & 7 & 9 & \\
\hline $7(3)$ & 2 & 9 & 9 & $\operatorname{PSL}(2,8)$ \\
\hline $8(3)$ & 3 & 3 & 7 & $\operatorname{PSL}(2,8) \times 2$ \\
\hline $9(3)$ & 3 & 3 & 9 & $\operatorname{PSL}(2,8) \times 2$ \\
\hline $10(3)$ & 3 & 7 & 7 & $\operatorname{PSL}(2,8)$ \\
$11(3)$ & 3 & 7 & 7 & $\operatorname{PSL}(2,8) \times 2$ \\
\hline $12-13(3)$ & 3 & 7 & 9 & \\
\hline $14(3)$ & 3 & 9 & 9 & $\operatorname{PSL}(2,8) \times 2$ \\
\hline $15(3)$ & 7 & 7 & 7 & $\operatorname{PSL}(2,8) \times 2$ \\
$16(3)$ & 7 & 7 & 7 & $\operatorname{PSL}(2,8) \times \operatorname{Sym}(3)$ \\
\hline $17-18(3)$ & 7 & 7 & 9 & $\operatorname{PSL}(2,8)$ \\
$19(3)$ & 7 & 7 & 9 & $\operatorname{PSL}(2,8) \times 2$ \\
\hline $20-21(3)$ & 7 & 9 & 9 & $\operatorname{PSL}(2,8)$ \\
$22-23(3)$ & 7 & 9 & 9 & $\operatorname{PSL}(2,8) \times 2$ \\
\hline $24(3)$ & 9 & 9 & 9 & $\operatorname{PSL}(2,8) \times \operatorname{Sym}(3)$ \\
\hline
\end{tabular}


3.5. $\operatorname{PSL}(\mathbf{2 , 1 1})$.

\begin{tabular}{||c|c|c|c|c||}
\hline Nr. & $1-2$ & $1-3$ & $2-3$ & $\operatorname{Cor}(\Gamma, G)$ \\
\hline $1(1)$ & 2 & 5 & 5 & $\operatorname{PGL}(2,11)$ \\
\hline $2(2)$ & 2 & 5 & 6 & \\
\hline $3(1)$ & 2 & 6 & 6 & $\operatorname{PGL}(2,11)$ \\
\hline $4(1)$ & 3 & 3 & 5 & $\operatorname{PGL}(2,11)$ \\
\hline $5-6(1)$ & 3 & 3 & 6 & $\operatorname{PGL}(2,11)$ \\
\hline $7(2)$ & 3 & 5 & 5 & $\operatorname{PSL}(2,11) \times 2$ \\
\hline $8-9(2)$ & 3 & 5 & 6 & \\
\hline $10(2)$ & 3 & 6 & 6 & $\operatorname{PSL}(2,11) \times 2$ \\
\hline $11(2)$ & 5 & 5 & 5 & $\operatorname{PSL}(2,11) \times 2$ \\
\hline $12-13(1)$ & 5 & 5 & 6 & $\operatorname{PGL}(2,11)$ \\
$14-15(2)$ & 5 & 5 & 6 & $\operatorname{PSL}(2,11)$ \\
\hline $16(1)$ & 5 & 6 & 6 & $\operatorname{PGL}(2,11)$ \\
$17(2)$ & 5 & 6 & 6 & $\operatorname{PSL}(2,11) \times 2$ \\
\hline
\end{tabular}

\begin{tabular}{||c|c|c|c|c|c|c|c|c||}
\hline Nr. & $1-2$ & $1-3$ & $1-4$ & $2-3$ & $2-4$ & $3-4$ & $\operatorname{Cor}(\Gamma, G)$ & $G_{0}, G_{1}, G_{2}, G_{3}$ \\
\hline $18(2)$ & 2 & 3 & 3 & 2 & 5 & 5 & $\operatorname{PSL}(2,11)$ & $A_{5}, A_{5}, A_{5}, D_{12}$ \\
$19(2)$ & 2 & 3 & 3 & 2 & 5 & 5 & $\operatorname{PSL}(2,11) \times 2$ & $A_{5}, A_{5}, A_{5}, D_{12}$ \\
\hline $20(1)$ & 2 & 2 & 3 & 3 & 5 & 2 & $\operatorname{PGL}(2,11)$ & $A_{5}, A_{5}, D_{12}, D_{12}$ \\
\hline $21(1)$ & 5 & 5 & 3 & 3 & 5 & 5 & $\operatorname{PSL}(2,11): \mathrm{D}_{8}$ & $A_{5}, A_{5}, A_{5}, A_{5}$ \\
\hline
\end{tabular}

3.6. $\mathbf{M}_{11}$.

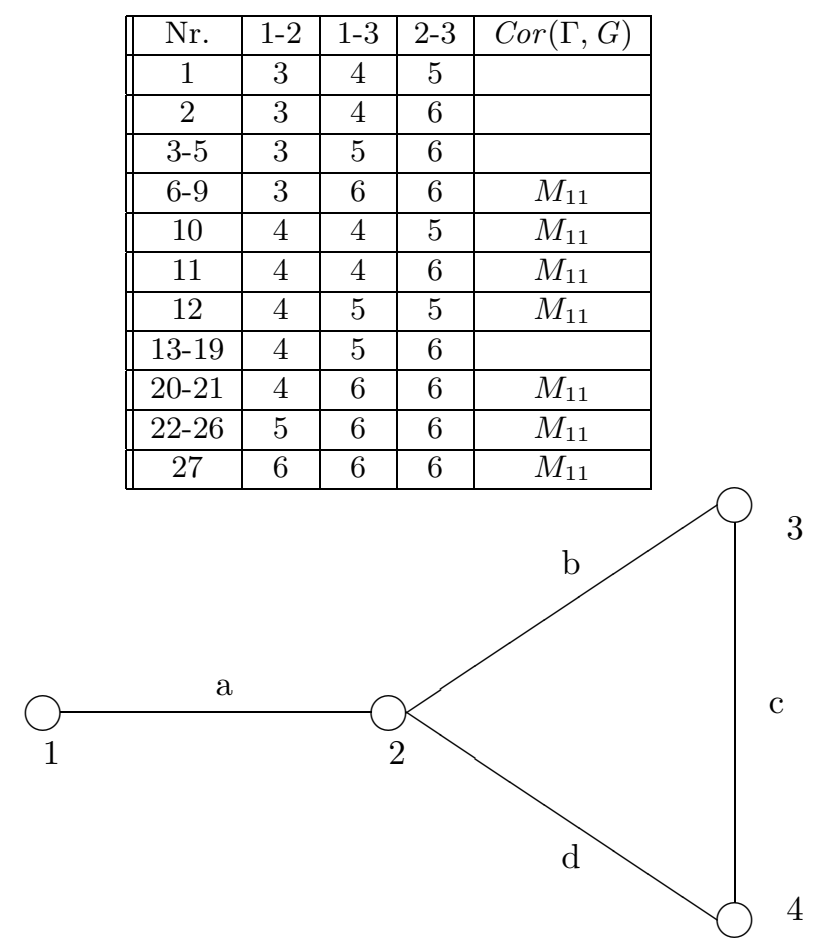

The next table is read as follows. The first column gives a number to each geometry. There is no number in parentheses anymore, because $\operatorname{Aut}\left(M_{11}\right)=M_{11}$ and thus $M_{11}$ cannot fuse any of its conjugacy classes of geometries. The next columns, labelled $a, b, c$, and $d$, give the parameters to put on the diagram given above, where there are also labels $a, b, c$, and $d$. The column labelled $\operatorname{Cor}(\Gamma, G)$ gives 
the correlation group and the column $G_{0}, G_{1}, G_{2}, G_{3}$ gives the maximal parabolic subgroups of the geometry. The first one corresponds to the node labelled "1" on the diagram above, etc.

\begin{tabular}{||c|c|c|c|c|c|c||}
\hline Nr. & a & b & c & d & $\operatorname{Cor}(\Gamma, G)$ & $G_{0}, G_{1}, G_{2}, G_{3}$ \\
\hline 28 & 3 & 3 & 3 & 5 & & $P S L(2,11), D_{12}, A_{5}, S_{4}$ \\
\hline 29 & 3 & 3 & 3 & 6 & & $P S L(2,11), D_{12}, S_{3} \times S_{3}, S_{4}$ \\
\hline 30 & 3 & 5 & 3 & 6 & & $P S L(2,11), D_{12}, S_{3} \times S_{3}, A_{5}$ \\
\hline 31 & 4 & 3 & 3 & 4 & & $A_{6}, D_{12}, 3^{2}: D_{8}, S_{4}$ \\
\hline 32 & 4 & 3 & 3 & 5 & & $A_{6}, D_{12}, S_{5}, S_{4}$ \\
\hline 33 & 4 & 3 & 3 & 6 & & $P S L(2,11), D_{12}, 3^{2}: D_{8}, S_{4}$ \\
34 & 4 & 3 & 3 & 6 & & $P S L(2,11), D_{12}, S_{5}, S_{4}$ \\
\hline 35 & 4 & 4 & 3 & 5 & & $A_{6}, D_{12}, S_{5}, 3^{2}: D_{8}$ \\
\hline 36 & 4 & 6 & 3 & 6 & $M_{11}$ & $P S L(2,11), D_{12}, 3^{2}: D_{8}, S_{5}$ \\
\hline 37 & 5 & 3 & 3 & 4 & & $G L(2,3), D_{12}, S_{5}, A_{5}$ \\
\hline 38 & 5 & 3 & 3 & 6 & & $G L(2,3), D_{12}, P S L(2,11), A_{5}$ \\
\hline 39 & 5 & 5 & 3 & 6 & & $P S L(2,11), D_{12}, S_{5}, A_{5}$ \\
\hline 40 & 6 & 3 & 3 & 6 & & $P S L(2,11), D_{12}, S_{5}, S_{3} \times S_{3}$ \\
\hline 41 & 6 & 4 & 3 & 4 & $M_{11}$ & $S_{5}, D_{12}, 3^{2}: D_{8}, S_{5}$ \\
\hline 42 & 6 & 4 & 3 & 5 & & $A_{6}, D_{12}, S_{5}, 3^{2}: D_{8}$ \\
\hline
\end{tabular}

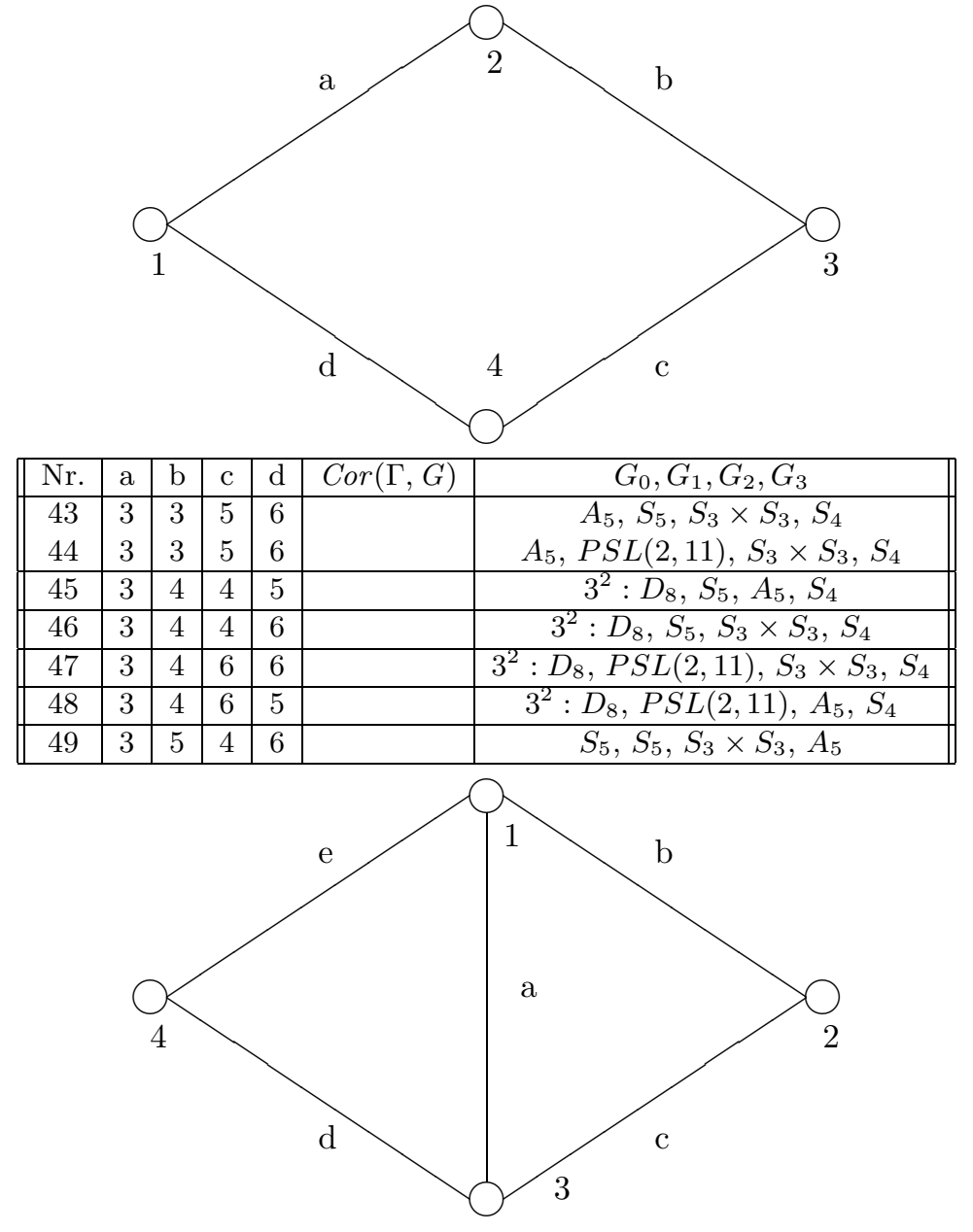




\begin{tabular}{|c|c|c|c|c|c|c|c|}
\hline Nr. & $\mathrm{a}$ & b & $\mathrm{c}$ & $\mathrm{d}$ & $\mathrm{e}$ & $\operatorname{Cor}(\Gamma, G)$ & $G_{0}, G_{1}, G_{2}, G_{3}$ \\
\hline 50 & 3 & 3 & 3 & 5 & 6 & & $A_{5}, P S L(2,11), S_{3} \times S_{3}, S_{4}$ \\
\hline 51 & 3 & 3 & 4 & 3 & 5 & & $S_{4}, P S L(2,11), A_{5}, G L(2,3)$ \\
\hline 52 & 3 & 3 & 4 & 3 & 6 & & $S_{4}, P S L(2,11), S_{3} \times S_{3}, G L(2,3)$ \\
\hline 53 & 3 & 3 & 4 & 4 & 4 & & $3^{2}: D_{8}, S_{5}, S_{4}, G L(2,3)$ \\
\hline 54 & 3 & 3 & 4 & 4 & 5 & & $3^{2}: D_{8}, A_{6}, A_{5}, G L(2,3)$ \\
\hline 55 & 3 & 3 & 4 & 6 & 3 & & $S_{4}, P S L(2,11), 3^{2}: D_{8}, G L(2,3)$ \\
\hline 56 & 3 & 3 & 5 & 3 & 6 & & $A_{5}, P S L(2,11), S_{3} \times S_{3}, G L(2,3)$ \\
\hline 57 & 3 & 3 & 6 & 4 & 5 & & $3^{2}: D_{8}, A_{6}, A_{5}, G L(2,3)$ \\
\hline 58 & 3 & 3 & 6 & 5 & 5 & & $S_{5}, A_{6}, A_{5}, G L(2,3)$ \\
\hline 59 & 3 & 3 & 6 & 6 & 4 & & $P S L(2,11), S_{5}, S_{4}, G L(2,3)$ \\
\hline 60 & 3 & 3 & 6 & 6 & 6 & & $S_{5}, P S L(2,11), S_{3} \times S_{3}, G L(2,3)$ \\
\hline 61 & 3 & 4 & 4 & 6 & 4 & & $3^{2}: D_{8}, S_{5}, 3^{2}: D_{8}, S_{5}$ \\
\hline 62 & 3 & 4 & 6 & 3 & 5 & & $S_{3} \times S_{3}, A_{5}, S_{5}, S_{5}$ \\
\hline 63 & 3 & 4 & 6 & 4 & 6 & $M_{11} \times 2$ & $3^{2}: D_{8}, S_{5}, 3^{2}: D_{8}, S_{5}$ \\
\hline 64 & 4 & 3 & 3 & 3 & 5 & & $S_{4}, A_{6}, A_{5}, G L(2,3)$ \\
\hline 65 & 4 & 3 & 4 & 3 & 5 & & $S_{4}, A_{6}, A_{5}, 3^{2}: D_{8}$ \\
\hline 66 & 4 & 3 & 6 & 4 & 5 & & $P S L(2,11), S_{4}, S_{3} \times S_{3}, S_{5}$ \\
\hline 67 & 5 & 3 & 3 & 6 & 3 & & $S_{3} \times S_{3}, P S L(2,11), S_{4}, S_{3} \times S_{3}$ \\
\hline 68 & 5 & 3 & 4 & 4 & 4 & & $3^{2}: D_{8}, S_{5}, S_{4}, A_{6}$ \\
\hline 69 & 5 & 3 & 5 & 6 & 3 & & $S_{5}, P S L(2,11), S_{4}, A_{5}$ \\
\hline 70 & 5 & 4 & 4 & 4 & 6 & & $A_{6}, S_{5}, 3^{2}: D_{8}, 3^{2}: D_{8}$ \\
\hline 71 & 6 & 3 & 4 & 3 & 6 & & $S_{4}, P S L(2,11), S_{3} \times S_{3}, S_{5}$ \\
\hline 72 & 6 & 3 & 5 & 5 & 6 & & $A_{5}, S_{5}, S_{3} \times S_{3}, P S L(2,11)$ \\
\hline
\end{tabular}

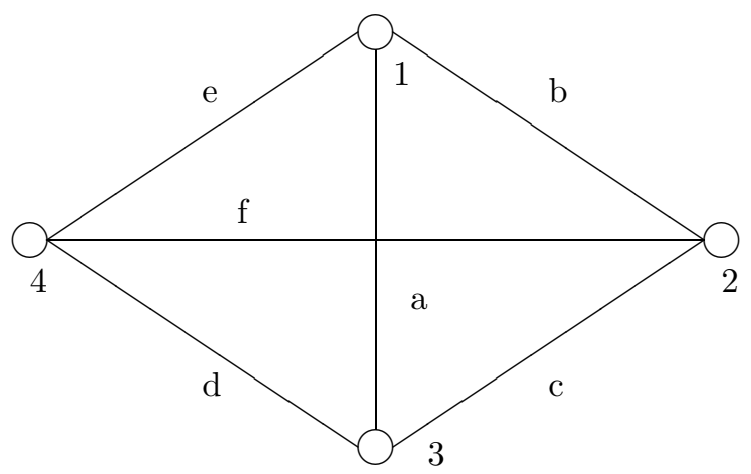

\begin{tabular}{||c|c|c|c|c|c|c|c|c||}
\hline Nr. & a & b & c & d & e & $\mathrm{f}$ & $\operatorname{Cor}(\Gamma, G)$ & $G_{0}, G_{1}, G_{2}, G_{3}$ \\
\hline 73 & 3 & 3 & 5 & 6 & 3 & 6 & $M_{11} \times 2$ & $P S L(2,11), G L(2,3), G L(2,3), A_{5}$ \\
\hline 74 & 3 & 3 & 4 & 3 & 5 & 4 & $M_{11}$ & $3^{2}: D_{8}, A_{5}, P S L(2,11), G L(2,3)$ \\
\hline 75 & 3 & 3 & 4 & 4 & 3 & 5 & $M_{11} \times 2$ & $S_{5}, G L(2,3), A_{5}, G L(2,3)$ \\
\hline
\end{tabular}

\section{Other GROUPS}

4.1. Alt(4). There is no thin, residually connected geometry on which Alt(4) acts flag-transitively.

4.2. Alt (4) $\times 2$. There is no thin, residually connected geometry on which $\operatorname{Alt}(4) \times$ 2 acts flag-transitively. 


\section{3. $\operatorname{Sym}(4)$.}

\begin{tabular}{||c|c|c|c|c||}
\hline Nr. & $1-2$ & $1-3$ & $2-3$ & $\operatorname{Cor}(\Gamma, G)$ \\
\hline $1(1)$ & 2 & 3 & 3 & $\operatorname{Sym}(4) \times 2$ \\
\hline $2(1)$ & 2 & 3 & 4 & \\
\hline $3(1)$ & 3 & 3 & 3 & $\operatorname{Sym}(4) \times \operatorname{Sym}(3)$ \\
\hline
\end{tabular}

4.4. $\operatorname{Alt}(5) \times 2$.

\begin{tabular}{||c|c|c|c|c||}
\hline Nr. & $1-2$ & $1-3$ & $2-3$ & $\operatorname{Cor}(\Gamma, G)$ \\
\hline $1(2)$ & 2 & 3 & 5 & \\
\hline $2-3(2)$ & 2 & 3 & 10 & \\
\hline $4(1)$ & 2 & 5 & 5 & $\operatorname{Sym}(5) \times 2$ \\
\hline $5-6(2)$ & 2 & 5 & 6 & \\
\hline $7-8(2)$ & 2 & 5 & 10 & \\
\hline $9(2)$ & 3 & 3 & 5 & $\operatorname{Alt}(5) \times 2^{2}$ \\
\hline $10(2)$ & 3 & 5 & 5 & $\operatorname{Alt}(5) \times 2^{2}$ \\
$11(1)$ & 3 & 5 & 5 & $\operatorname{Sym}(5) \times 2$ \\
\hline $12(2)$ & 5 & 5 & 5 & $\operatorname{Alt}(5) \times 2 \times \operatorname{Sym}(3)$ \\
\hline
\end{tabular}

\begin{tabular}{||c|c|c|c|c|c|c|c|c||}
\hline Nr. & $1-2$ & $1-3$ & $1-4$ & $2-3$ & $2-4$ & $3-4$ & $\operatorname{Cor}(\Gamma, G)$ & $G_{0}, G_{1}, G_{2}, G_{3}$ \\
\hline $13(2)$ & 2 & 2 & 2 & 2 & 3 & 5 & & $A_{5}, D_{20}, D_{12}, 2^{3}$ \\
\hline $14(1)$ & 2 & 2 & 2 & 2 & 5 & 5 & $\operatorname{Sym}(5) \times 2$ & $A_{5}, D_{20}, D_{20}, 2^{3}$ \\
\hline $15(2)$ & 2 & 2 & 2 & 3 & 3 & 5 & $\operatorname{Alt}(5) \times 2^{2}$ & $A_{5}, D_{20}, D_{12}, D_{12}$ \\
\hline $16(2)$ & 2 & 2 & 2 & 3 & 5 & 5 & $\operatorname{Alt}(5) \times 2^{2}$ & $A_{5}, D_{20}, D_{20}, D_{12}$ \\
\hline
\end{tabular}

\section{5. $\operatorname{Sym}(5)$.}

\begin{tabular}{||c|c|c|c|c||}
\hline Nr. & $1-2$ & $1-3$ & $2-3$ & $\operatorname{Cor}(\Gamma, G)$ \\
\hline $1(1)$ & 2 & 4 & 5 & \\
\hline $2(1)$ & 2 & 4 & 6 & \\
\hline $3(1)$ & 2 & 5 & 6 & \\
\hline $4(1)$ & 2 & 6 & 6 & $\operatorname{Sym}(5) \times 2$ \\
\hline $5(1)$ & 3 & 4 & 4 & $\operatorname{Sym}(5) \times 2$ \\
\hline $6-7(1)$ & 3 & 4 & 6 & \\
\hline $8(1)$ & 4 & 4 & 5 & $\operatorname{Sym}(5) \times 2$ \\
\hline $9(1)$ & 4 & 5 & 6 & \\
\hline $10(1)$ & 5 & 6 & 6 & $\operatorname{Sym}(5) \times 2$ \\
\hline
\end{tabular}

\begin{tabular}{||c|c|c|c|c|c|c|c|c||}
\hline Nr. & $1-2$ & $1-3$ & $1-4$ & $2-3$ & $2-4$ & $3-4$ & $\operatorname{Cor}(\Gamma, G)$ & $G_{0}, G_{1}, G_{2}, G_{3}$ \\
\hline $11(1)$ & 2 & 2 & 3 & 3 & 3 & 3 & $\operatorname{Sym}(5) \times 2$ & $S_{4}, S_{4}, S_{4}, D_{12}$ \\
\hline $12(1)$ & 3 & 2 & 2 & 2 & 3 & 3 & $\operatorname{Sym}(5) \times 2$ & $S_{4}, D_{12}, S_{4}, D_{12}$ \\
\hline $13(1)$ & 3 & 3 & 3 & 3 & 3 & 3 & $\operatorname{Sym}(5) \times \operatorname{Sym}(4)$ & $S_{4}, S_{4}, S_{4}, S_{4}$ \\
\hline
\end{tabular}


4.6. $\operatorname{Sym}(5) \times 2$.

\begin{tabular}{||c|c|c|c|c||}
\hline Nr. & $1-2$ & $1-3$ & $2-3$ & $\operatorname{Cor}(\Gamma, G)$ \\
\hline $1-2$ & 2 & 4 & 5 & \\
\hline $3-8$ & 2 & 4 & 6 & \\
\hline $9-12$ & 2 & 4 & 10 & \\
\hline $13-14$ & 2 & 5 & 6 & \\
\hline $15-16$ & 2 & 6 & 6 & $\operatorname{Sym}(5) \times 2^{2}$ \\
$17-18$ & 2 & 6 & 6 & $\operatorname{Sym}(5) \times 2$ \\
\hline $19-22$ & 2 & 6 & 10 & \\
\hline $23-24$ & 3 & 4 & 4 & $\operatorname{Sym}(5) \times 2^{2}$ \\
\hline $25-28$ & 3 & 4 & 6 & \\
\hline $29-30$ & 3 & 6 & 6 & $\operatorname{Sym}(5) \times 2^{2}$ \\
\hline $31-32$ & 4 & 4 & 5 & $\operatorname{Sym}(5) \times 2^{2}$ \\
\hline $33-34$ & 4 & 4 & 6 & $\operatorname{Sym}(5) \times 2$ \\
\hline $35-36$ & 4 & 4 & 10 & $\operatorname{Sym}(5) \times 2$ \\
\hline $37-40$ & 4 & 5 & 6 & \\
\hline $41-48$ & 4 & 6 & 6 & $\operatorname{Sym}(5) \times 2$ \\
\hline $49-52$ & 4 & 6 & 10 & \\
\hline $53-54$ & 5 & 6 & 6 & $\operatorname{Sym}(5) \times 2^{2}$ \\
\hline $55-56$ & 6 & 6 & 10 & $\operatorname{Sym}(5) \times 2$ \\
\hline
\end{tabular}

\begin{tabular}{||c|c|c|c|c|c|c|c|c||}
\hline Nr. & $1-2$ & $1-3$ & $1-4$ & $2-3$ & $2-4$ & $3-4$ & $\operatorname{Cor}(\Gamma, G)$ & $G_{0}, G_{1}, G_{2}, G_{3}$ \\
\hline $57-58(1)$ & 2 & 2 & 2 & 2 & 4 & 5 & & $S_{5}, D_{20}, 2 \times D_{8}, 2^{3}$ \\
\hline $59-60(1)$ & 2 & 2 & 2 & 2 & 4 & 6 & & $S_{5}, 2^{2} \times S_{3}, 2 \times D_{8}, 2^{3}$ \\
\hline $61-62(1)$ & 2 & 2 & 2 & 2 & 5 & 6 & & $S_{5}, 2^{2} \times S_{3}, D_{20}, 2^{3}$ \\
\hline $63-64(1)$ & 2 & 2 & 2 & 2 & 6 & 6 & $\operatorname{Sym}(5) \times 2^{2}$ & $S_{5}, 2^{2} \times S_{3}, 2^{2} \times S_{3}, 2^{3}$ \\
\hline $65-66(1)$ & 2 & 2 & 2 & 3 & 4 & 4 & $\operatorname{Sym}(5) \times 2^{2}$ & $S_{5}, 2 \times D_{8}, 2 \times D_{8}, D_{12}$ \\
\hline $67-70(1)$ & 2 & 2 & 2 & 3 & 4 & 6 & & $S_{5}, 2^{2} \times S_{3}, 2 \times D_{8}, D_{12}$ \\
\hline $71-72(1)$ & 2 & 2 & 2 & 4 & 4 & 5 & $\operatorname{Sym}(5) \times 2^{2}$ & $S_{5}, D_{20}, 2 \times D_{8}, 2 \times D_{8}$ \\
\hline $73-74(1)$ & 2 & 2 & 2 & 4 & 5 & 6 & & $S_{5}, 2^{2} \times S_{3}, D_{20}, 2 \times D_{8}$ \\
\hline $75-76(1)$ & 2 & 2 & 2 & 5 & 6 & 6 & $\operatorname{Sym}(5) \times 2^{2}$ & $S_{5}, 2^{2} \times S_{3}, 2^{2} \times S_{3}, D_{20}$ \\
\hline $77-78(1)$ & 2 & 2 & 6 & 3 & 3 & 3 & $\operatorname{Sym}(5) \times 2^{2}$ & $S_{4}, 2 \times S_{4}, 2 \times S_{4}, D_{12}$ \\
\hline $79-80(1)$ & 3 & 2 & 2 & 3 & 2 & 6 & & $2 \times S_{4}, 2 \times D_{12}, D_{12}, S_{4}$ \\
\hline $81(1)$ & 3 & 2 & 2 & 6 & 2 & 3 & $\operatorname{Sym}(5) \times 2^{2}$ & $2 \times S_{4}, D_{12}, D_{12}, 2 \times S_{4}$ \\
\hline
\end{tabular}

\begin{tabular}{||c|c|c|c|c|c|c|c|c|c|c|c||}
\hline Nr. & $1-2$ & $1-3$ & $1-4$ & $1-5$ & $2-3$ & $2-4$ & $2-5$ & $3-4$ & $3-5$ & $4-5$ & $\operatorname{Cor}(\Gamma, G)$ \\
\hline $82-83$ & 2 & 2 & 2 & 2 & 2 & 2 & 3 & 3 & 3 & 3 & $\operatorname{Sym}(5) \times 2^{2}$ \\
\hline $84-85$ & 2 & 2 & 2 & 2 & 3 & 3 & 3 & 3 & 3 & 3 & $\operatorname{Sym}(5) \times 2 \times \operatorname{Sym}(4)$ \\
\hline $86-87$ & 2 & 2 & 2 & 2 & 3 & 2 & 2 & 3 & 2 & 3 & $\operatorname{Sym}(5) \times 2^{2}$ \\
\hline
\end{tabular}

The parabolic subgroups of geometries 82 to 87 can be guessed easily by looking at those of $\operatorname{Sym}(5)$. 


\section{7. $\operatorname{PGL}(2,7)$.}

\begin{tabular}{||c|c|c|c|c||}
\hline Nr. & $1-2$ & $1-3$ & $2-3$ & $\operatorname{Cor}(\Gamma, G)$ \\
\hline $1(1)$ & 2 & 3 & 7 & \\
\hline $2-3(1)$ & 2 & 3 & 8 & \\
\hline $4(1)$ & 2 & 4 & 6 & \\
\hline $5(1)$ & 2 & 4 & 7 & \\
\hline $6-7(1)$ & 2 & 4 & 8 & \\
\hline $8(1)$ & 2 & 6 & 6 & $\operatorname{PGL}(2,7) \times 2$ \\
\hline $9(1)$ & 2 & 6 & 7 & \\
\hline $10-11(1)$ & 2 & 6 & 8 & \\
\hline $12(1)$ & 2 & 7 & 7 & $\operatorname{PGL}(2,7) \times 2$ \\
\hline $13-14(1)$ & 2 & 7 & 8 & \\
\hline $15-16(1)$ & 2 & 8 & 8 & $\operatorname{PGL}(2,7) \times 2$ \\
\hline $17(1)$ & 3 & 3 & 4 & $\operatorname{PGL}(2,7) \times 2$ \\
\hline $18(1)$ & 3 & 3 & 7 & $\operatorname{PGL}(2,7) \times 2$ \\
\hline $19-20(1)$ & 3 & 4 & 7 & \\
\hline $21(1)$ & 3 & 6 & 6 & $\operatorname{PGL}(2,7) \times 2$ \\
\hline $22-24(1)$ & 3 & 6 & 8 & \\
\hline $25-26(1)$ & 3 & 7 & 7 & $\operatorname{PGL}(2,7) \times 2$ \\
\hline $27-29(1)$ & 3 & 8 & 8 & $\operatorname{PGL}(2,7) \times 2$ \\
$30-31(1)$ & 3 & 8 & 8 & $\operatorname{PGL}(2,7)$ \\
\hline $32(1)$ & 4 & 4 & 4 & $\operatorname{PGL}(2,7) \times \operatorname{Sym}(3)$ \\
\hline $33(1)$ & 4 & 4 & 7 & $\operatorname{PGL}(2,7) \times 2$ \\
\hline $34-35(1)$ & 4 & 6 & 6 & $\operatorname{PGL}(2,7) \times 2$ \\
\hline $36(1)$ & 4 & 6 & 8 & \\
\hline $37-38(1)$ & 4 & 7 & 7 & $\operatorname{PGL}(2,7) \times 2$ \\
\hline $39(1)$ & 4 & 8 & 8 & $\operatorname{PGL}(2,7) \times 2$ \\
\hline $40-42(1)$ & 6 & 7 & 8 & \\
\hline $43(1)$ & 7 & 7 & 7 & $\operatorname{PGL}(2,7) \times \operatorname{Sym}(3)$ \\
\hline $44(1)$ & 7 & 8 & 8 & $\operatorname{PGL}(2,7)$ \\
\hline
\end{tabular}

4.8. $\mathbf{M}_{10}$. There is no thin, residually connected geometry on which $M_{10}$ acts flagtransitively.

\section{Applications}

The aim of this section is to show that building such an atlas may be useful, not only to make a collection of geometries, but also to find theorems and to solve open problems. The following theorems tend to show that for a given diagram, we can determine a lower bound on the order of a group $G$ for it to act flag-transitively on the geometry corresponding to the diagram. Sometimes this lower bound is reached and we can see it by looking at our collection of geometries given in the two previous sections. This means we can say that such a group is (one of) the smallest group(s) acting regularly on such a diagram. Sometimes we can even prove that the group for which we have an example is the only one having this order.

Theorem 2. The group Alt(5) is the smallest group that acts regularly on the following diagram:

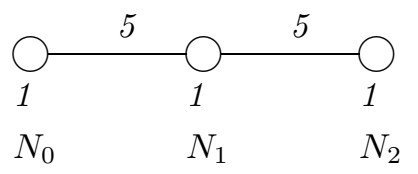


Proof. Looking at the diagram, we know that $|G|=N_{0} \cdot 10=N_{1} \cdot 4=N_{2} \cdot 10=20 \cdot \epsilon$. Thus $N_{0}=2 \cdot \epsilon_{0}$. But $N_{0} \geq 5$ and so $N_{0} \geq 6$. So $\epsilon \geq 3$. This means that the order of $G$ is at least 60 . Looking at the thin geometries of $A l t(5)$ given in section 3.1 , we see that geometry number 2 has the diagram we are looking at. Thus there exists a group $G$ whose order is 60 and which acts regularly on this diagram. Now we still have to show that it is the only group of order 60 that does so. Looking at all the groups of order 60 (they are implemented in the computer algebra package MAGma [13]), we find only four groups that have at least two subgroups isomorphic to $D_{10}$ and one isomorphic to $D_{4}$. They are Alt(5), $D_{60}, 6 \times D_{10}$ and $D_{6} \times D_{10}$. An exhaustive search of all the thin rank 3 geometries of these groups shows that $\operatorname{Alt}(5)$ is the only one to have a geometry with this diagram.

Theorem 3. The group Alt $(5) \times 2$ is the smallest group that acts regularly on the following diagram:

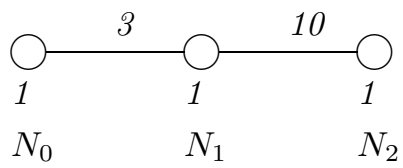

Proof. Looking at the diagram, we know that $|G|=N_{0} \cdot 20=N_{1} \cdot 4=N_{2} \cdot 6=60 \cdot \epsilon$. This means that the order of $G$ is at least 60 . There are only two groups of order 60 which have subgroups isomorphic to $D_{20}, D_{4}$ and $D_{6}$. They are $D_{60}$ and $D_{6} \times D_{10}$. Using our set of programs, it is easy to construct all the thin geometries on which these two groups act regularly. This shows us that neither of these two groups acts regularly on the diagram given above. Thus we can conclude that $|G|>60$ and hence $|G| \geq 120$. Then, looking at the geometries of $\operatorname{Alt}(5) \times 2$, we see that geometries 2 and 3 have the diagram we are looking for. We still have to show that it is the only group of order 120 that acts regularly on this diagram. To do so, we use the database of all small groups of order less than 1000 available in Gap [21]. There are 47 such groups. Eighteen of them have subgroups isomorphic to $D_{4}, D_{6}$ and $D_{20}$. We then use our set of programs to check that only one of these groups, namely, $\operatorname{Alt}(5) \times 2$, has such a thin geometry.

Theorem 4. The groups Alt(5) $\times 2$ and Sym(5) are the smallest groups that act regularly on the following diagram:

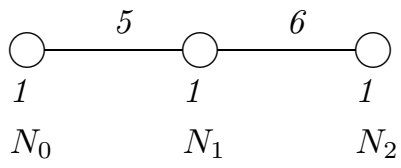

Proof. Looking at the diagram, we know that $|G|=N_{0} \cdot 12=N_{1} \cdot 4=N_{2} \cdot 10=60 \cdot \epsilon$. Thus the order of $G$ is at least 60 . There are only two groups of order 60 which have subgroups isomorphic to $D_{10}, D_{4}$ and $D_{12}$. They are $D_{60}$ and $D_{6} \times D_{10}$. Using our set of programs, it is easy to construct all the thin geometries on which these two groups act regularly. This shows us that neither of these two groups acts regularly on the diagram given above. Thus we can conclude that $|G|>60$ and hence $|G| \geq 120$. This diagram appears for geometries 5 and 6 of $\operatorname{Alt}(5) \times 2$ and 3 of $\operatorname{Sym}(5)$. To check that thses are the only two groups acting regularly on that diagram, we do the same work as in the previous theorem. Out of the 47 groups of order 120 , there are only 19 of them that have subgroups isomorphic to $D_{4}, D_{10}$ 
and $D_{12}$. Then we check with our programs that only $\operatorname{Alt}(5) \times 2$ and $\operatorname{Sym}(5)$ can act regularly on that diagram.

Theorem 5. The groups $2 \times$ Sym(4) and $D_{6} \times D_{8}$ are the smallest groups that act regularly on the following diagram. The group $P 9 N 2=3^{2}: D_{8}$ appearing in [11] is the next one.

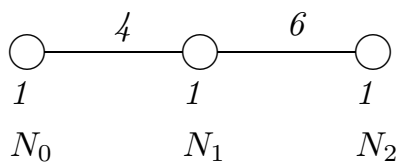

Proof. Because $N_{0} \cdot 12=N_{1} \cdot 4=N_{2} \cdot 8=|G|$, we know that $|G|=24 \cdot n$ where $n$ is an integer. Also, the diagram tells us that $N_{0} \geq 4$. Thus, $|G|=24 \cdot n \geq 48$. Looking at all the non-abelian groups of order 48, we get 12 groups that have subgroups isomorphic to $D_{8}, D_{4}$, and $D_{12}$. Using our set of programs, we see that $2 \times \operatorname{Sym}(4)$ and $D_{6} \times D_{8}$ are the only two groups that act regularly on the diagram given above.

Now, by looking at geometry number 41 of $M_{11}$, we see that the residue of $3^{2}: D_{8}$ has the diagram given above. And this $3^{2}: D_{8}$ is the primitive group appearing in [11] as group $P 9 N 2$. Thus there exists a group of order 72 that acts regularly on this diagram. We still have to show that it is the only one. To do so, we look at all the non-abelian groups of order 72 . There are 44 such groups. Only 11 of them have some of their subgroups isomorphic to $D_{4}, D_{8}$, and $D_{12}$ which are the three parabolic subgroups of our geometry. We then construct all their thin geometries using our set of programs. And it turns out that no other group of order 72 has such a geometry.

Theorem 6. The group $D_{10} \times D_{10}$ is the smallest group that acts regularly on the following diagram. The group Alt $(5) \times 2$ is the next one.

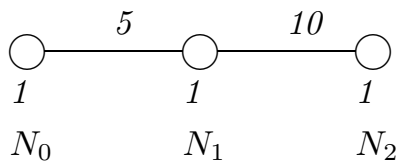

Proof. Because $N_{0} \cdot 20=N_{1} \cdot 4=N_{2} \cdot 10=|G|$, we know that $|G|=20 \cdot n$ where $n$ is an integer. Also, the diagram tells us that $N_{2} \geq 10$. Thus, $|G|=20 \cdot n \geq 100$. Looking at all the non-abelian groups of order 100, we get 4 groups that have subgroups isomorphic to $D_{10}, D_{4}$, and $D_{20}$. Using our set of programs, we see that $D_{10} \times D_{10}$ is the only one that acts regularly on the diagram given above.

The next possible size for $G$ is 120 . Looking at the geometries obtained for $\operatorname{Alt}(5) \times 2$, we see that two of them, namely geometries number 7 and 8 , have the corresponding diagram. Then, looking at all the groups of order 120, we know that 13 of them have subgroups isomorphic to $D_{4}, D_{10}$ and $D_{20}$. None of them, except Alt $(5) \times 2$ acts regularly on the diagram given above.

Theorem 7. The group

$$
\begin{aligned}
& G=\langle(1,18,23,9,16,21,7,14,29,5,12,27,3,20,25) \\
& (2,17,24,10,15,22,8,13,30,6,11,28,4,19,26) \\
& (1,13)(2,14)(3,11)(4,12)(5,19)(6,20)(7,17)(8,18)(9,15) \\
& (10,16)(21,23)(22,24)(25,29)(26,30)\rangle
\end{aligned}
$$


of order 120 and of structure $(5 \times$ Alt $(4)): 2$, is the smallest group that acts regularly on the following diagram:

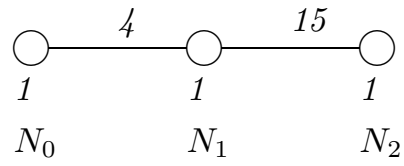

Proof. Because $N_{0} \cdot 30=N_{1} \cdot 4=N_{2} \cdot 8=|G|$, we know that $|G|=120 \cdot n$, where $n$ is an integer. While scanning all groups of order 120 having subgroups isomorphic to $D_{4}, D_{6}$ and $D_{20}$, for the proof of Theorem 3, we found this group acting on the diagram given above. Looking at all the groups of order 120, we get 12 groups that have subgroups isomorphic to $D_{4}, D_{8}$, and $D_{30}$. Using our set of programs, we see that the group $G$ given here is the only one that acts regularly on the diagram given above.

Theorem 8. The group $D_{12} \times D_{10}$ is the smallest group that acts regularly on the following diagram:

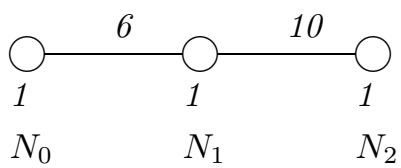

Proof. Because $N_{0} \cdot 20=N_{1} \cdot 4=N_{2} \cdot 12=|G|$, we know that $|G|=60 \cdot n$, where $n$ is an integer. Also, we see that $N_{2} \geq 10$, which implies $|G| \geq 120$. While scanning all groups of order 120 having subgroups isomorphic to $D_{4}, D_{6}$ and $D_{20}$, for the proof of Theorem 3, we found this group acting on the diagram given above. Looking at all the groups of order 120, we get 29 groups that have subgroups isomorphic to $D_{4}, D_{12}$, and $D_{20}$. Using our set of programs, we see that $2 \times D_{60}$ is the only one that acts regularly on the diagram given above.

\section{Thin GeOMETRIES AND REFLEXIVE MAPS}

According to [17], a map is the decomposition of an unbounded surface into $N_{2}$ non-overlapping simply-connected regions called faces by $N_{1}$ arcs called edges joining pairs of $N_{0}$ points called vertices. An automorphism of a map is a permutation of its elements preserving the relations of incidence. The automorphisms clearly form a group, called the group of the map. A map is regular if its group contains two particular automorphisms: one, say $R$, which cyclically permutes the edges that are successive sides of one face, and another, say $S$, which cyclically permutes the successive edges meeting at one vertex of this face. A regular map is reflexive if there is an automorphism $R_{1}$ of the group which interchanges the two vertices incident to a given edge without interchanging the two faces incident to it. Finally, a map is said to be of type $\{p, q\}$ if $p$ edges belong to a face and $q$ to a vertex.

Starting from a thin regular geometry whose diagram is linear, it is easy to construct a reflexive map. 
Lemma 2. Let $\Gamma\left(G ; G_{0}, G_{1}, G_{2}\right)$ be a thin regular geometry of rank 3 over a linear diagram of the following type:

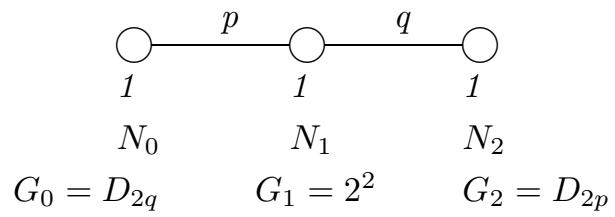

Then $\Gamma$ may be seen as a reflexive map $\mathcal{M}$ of type $\{p, q\}$.

Proof. The left cosets of $G_{0}$ (resp. $G_{1}, G_{2}$ ) are the vertices (resp. edges, faces) of $\mathcal{M}$. This map is clearly regular. When we fix an edge $e$ and a face $f$ incident to it, we take the residue of these two objects. It consists of the two vertices incident to the edge. The group stabilizing $e$ and $f$ is $G_{1} \cap G_{2}$, which is a cyclic group of order two. And it acts transitively on the two vertices. Thus the map is reflexive.

Now, starting from a regular map, it is easy to obtain a thin regular rank 3 geometry.

A string is a graph on a set $I$ such that we can order $I=\{1, \ldots, n\}$ so that the edges of the graph are $\{i, i+1\}, 1 \leq i \leq n$.

Lemma 3 ([1]). Let $G$ be a group, $I$ a finite set, and $\Gamma=\left(G_{i}: i \in I\right)$ a family of subgroups of $G$. Assume:

(i) for each subset $J$ of I of corank at least 2, $G_{J}=\left\langle G_{J \cup\{i\}}: i \in I \backslash J\right\rangle$, and

(ii) the connected components of the graph of the diagram of $\Gamma$ are strings. Then

(1) $G$ is flag-transitive on $\Gamma$.

(2) $\Gamma$ is residually connected.

Lemma 4. Let $\mathcal{M}=\{p, q\}$ be a reflexive map, and let $G$ be the group of the map. Then, there exists a regular geometry $\Gamma\left(G ; G_{0}, G_{1}, G_{2}\right)$ over a linear diagram of the following type:

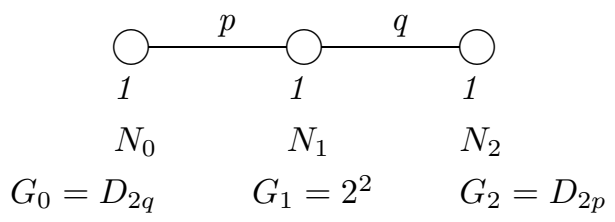

Proof. We simply take a vertex $v$, an edge $e$ and a face $f$ that are pairwise incident. Then $G_{0}$ (resp. $G_{1}, G_{2}$ ) is the stabilizer of $v$ (resp. $e, f$ ). Because $\mathcal{M}$ is a map, we have that $\Gamma\left(G ; G_{0}, G_{1}, G_{2}\right)$ is a thin geometry, and because its diagram is linear, we know thanks to Lemma 3 that the group $G$ acts flag-transitively on it.

\section{Petrie POLYGONS}

As in [17], 5.2, a Petrie polygon of a map is a "zig-zag" in which every two consecutive sides, but no three, belong to a face. In [17], 8.6, a method to compute the diameter of the Petrie polygons of a given map $\mathcal{M}=\{p, q\}$ is shown. Roughly speaking, if $\Gamma\left(G ; G_{0}, G_{1}, G_{2}\right)$ corresponds to the map $\{p, q\}$, then $G_{0} \cap G_{1}=\{\operatorname{Id}(G), a\}$, $G_{0} \cap G_{2}=\{I d(G), b\}$ and $G_{1} \cap G_{2}=\{I d(G), c\}$. The length of the Petrie polygon is then the order of the element $a \cdot b \cdot c$, that is, the smallest integer $r$ such that $(a \cdot b \cdot c)^{r}=\operatorname{Id}(G)$. The map is then denoted $\{p, q\}_{r}$. And a new map can be 
TABLE 1. The maps obtained in this atlas

\begin{tabular}{|c|c|c|c|c|c|c|c|}
\hline Map & Vertices & Edges & Faces & Group & Order & Smallest? & Reference \\
\hline$\{3,5\}_{5}$ & 6 & 15 & 10 & Alt (5) & 60 & Yes & {$[17]$} \\
\hline$\{3,7\}_{9}$ & 36 & 126 & 84 & $P S L(2,8)$ & 504 & $?$ & {$[17]$} \\
\hline$\{7,7\}_{9}$ & 36 & 126 & 36 & $P S L(2,8)$ & 504 & $?$ & \\
\hline$\{9,9\}_{9}$ & 28 & 126 & 28 & $P S L(2,8)$ & 504 & $?$ & \\
\hline$\{5,5\}_{5}$ & 66 & 165 & 66 & $P S L(2,11)$ & 660 & $?$ & [17] \\
\hline$\{5,6\}_{6}$ & 55 & 165 & 66 & $P S L(2,11)$ & 660 & $?$ & \\
\hline$\{3,3\}_{4}$ & 4 & 6 & 4 & $\operatorname{Sym}(4)$ & 24 & Yes & {$[17]$} \\
\hline$\{3,5\}_{10}$ & 12 & 30 & 20 & $\operatorname{Alt}(5) \times 2$ & 120 & Yes & {$[17]$} \\
\hline$\{3,10\}_{-}$ & 6 & 30 & 20 & $\operatorname{Alt}(5) \times 2$ & 120 & Yes & \\
\hline$\{5,5\}_{6}$ & 12 & 30 & 12 & $\operatorname{Alt}(5) \times 2$ & 120 & Yes & \\
\hline$\{5,6\}-$ & 10 & 30 & 12 & $\operatorname{Alt}(5) \times 2$ & 120 & Yes & \\
\hline$\{5,10\}_{-}$ & 6 & 30 & 12 & $\operatorname{Alt}(5) \times 2$ & 120 & No & \\
\hline$\{4,5\}_{6}$ & 12 & 30 & 15 & $\operatorname{Sym}(5)$ & 120 & Yes & \\
\hline$\{6,6\}_{6}$ & 10 & 30 & 10 & $\operatorname{Sym}(5)$ & 120 & $?$ & \\
\hline$\{4,5\}_{6}$ & 24 & 60 & 30 & $\operatorname{Sym}(5) \times 2$ & 240 & No & {$[17]$} \\
\hline$\{4,6\}_{10}$ & 20 & 60 & 30 & $\operatorname{Sym}(5) \times 2$ & 240 & $?$ & \\
\hline$\{6,6\}_{6}$ & 20 & 60 & 20 & $\operatorname{Sym}(5) \times 2$ & 240 & $?$ & \\
\hline$\{3,7\}_{8}$ & 24 & 84 & 56 & $P G L(2,7)$ & 336 & Yes & {$[17]$} \\
\hline$\{3,8\}_{8}$ & 21 & 84 & 56 & $P G L(2,7)$ & 336 & $?$ & \\
\hline$\{4,6\}_{8}$ & 28 & 84 & 42 & $P G L(2,7)$ & 336 & $?$ & \\
\hline$\{4,7\}_{8}$ & 24 & 84 & 42 & $P G L(2,7)$ & 336 & $?$ & \\
\hline$\{6,6\}_{8}$ & 28 & 84 & 28 & $P G L(2,7)$ & 336 & $?$ & \\
\hline$\{6,7\}_{7}$ & 24 & 84 & 28 & $P G L(2,7)$ & 336 & $?$ & \\
\hline$\{8,8\}_{8}$ & 21 & 84 & 21 & $P G L(2,7)$ & 336 & $?$ & \\
\hline$\{3,4\}_{6}$ & 6 & 12 & 8 & $\operatorname{Sym}(4) \times 2$ & 48 & Yes & [17] \\
\hline$\{4,6\}_{-}$ & 4 & 12 & 6 & $D_{6} \times D_{8}$ & 48 & Yes & \\
\hline$\{5,10\}_{-}$ & 5 & 25 & 10 & $D_{10} \times D_{10}$ & 100 & Yes & \\
\hline$\{4,15\}_{-}$ & 4 & 30 & 15 & See Th. 7 & 120 & Yes & \\
\hline
\end{tabular}

obtained by replacing the faces of the abstract polyhedron corresponding to the map, by the Petrie polygons. We then get a map $\{p, r\}_{q}$, which is called the Petrial of $\mathcal{M}$ in [28]. In that paper, the authors mention that sometimes problems occur and that the Petrial might not be a polyhedron anymore. We now give an example of such a situation. If we compute $r$ for geometries 2 and 3 of $\operatorname{Alt}(5) \times 2$, we obtain 5 and 10 . The value 5 is correct, and we see that $\{3,5\},\{3,10\}$ and $\{5,10\}$ are maps appearing as geometries of $\operatorname{Alt}(5) \times 2$. But because $\{10,10\}$ does not appear as a flag-transitive geometry of $A l t(5) \times 2$, we know that this value is not good.

Group explanation. To construct the Petrial of a polyhedron, we take the three generators $a, b$ and $c$, and we replace them by $a \cdot c, b$, and $c$. This gives a map of type $\{p, r\}$ most of the time. But sometimes, the subgroup $\langle a \cdot c, b\rangle \cap\langle b, c\rangle$ is not of order two. Thus the geometry obtained is not thin anymore. This is what happens for geometry 3 of $\operatorname{Alt}(5) \times 2$.

Geometric explanation. If we construct the incidence structure arising from geometry 3 of $\operatorname{Alt}(5) \times 2$, and if we try to construct the Petrie polygons of it, we see that there is always one vertex incident to four edges. Thus the Petrie polygons are not genuine polygons. They look locally as follows: 


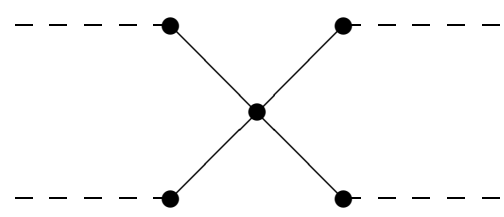

Then of course, if we replace the faces of geometry 3 of $\operatorname{Alt}(5) \times 2$ by these new faces, we do not have a polyhedron anymore.

Now we can take all the geometries with a linear diagram listed in the previous sections and convert them into reflexive maps. We then obtain new maps, and some of them are the smallest ones. Table 1 (on the previous page) lists all the maps obtained here. We only give one of the two or six related maps. It is well known that $\{p, q\}_{r},\{q, p\}_{r},\{p, r\}_{q},\{r, p\}_{q},\{q, r\}_{p}$ and $\{r, q\}_{p}$ have the same group acting on them. And if the Petrial of a given map is not a map anymore, we write "-" instead of $r$. Then, there are two maps that have the same group acting. They are $\{p, q\}_{-}$and $\{q, p\}_{-}$. We only mention one of them.

\section{REFERENCES}

1. M. Aschbacher, Flag structures on Tits geometries, Geom. Dedicata 14 (1983), 21-31. MR 84m:51010

2. F. Buekenhout, Diagrams for geometries and groups, J. Combin. Theory Ser. A 27 (1979), 121-151. MR 83f:51003

3. _,$\left(g, d, d^{*}\right)$-gons, Finite Geometries (Marcel Dekker, New York) (Johnson N.L., Kallaher M.J., and Long C.T., eds.), 1983, pp. 93-111. MR 84e:51013

4. F. Buekenhout (ed.), Handbook of incidence geometry, Elsevier, Amsterdam, 1995. MR 96e:51002

5. F. Buekenhout and P. Cara, Some properties of inductively minimal flag-transitive geometries, Bull. Belg. Math. Soc. 5 (1998), 213-219.

6. F. Buekenhout, P. Cara, and M. Dehon, Inductively minimal flag-transitive geometries, Mostly Finite Geometries (Johnson N.L., ed.), 1997, pp. 185-190. MR 88h:51020

7. _ Geometries of small almost simple groups based on maximal subgroups, Bull. Belg. Math. Soc. - Simon Stevin Suppl. (1998). CMP 98:12

8. F. Buekenhout, M. Dehon, and D. Leemans, All geometries of the Mathieu group $M_{11}$ based on maximal subgroups, Experiment. Math. 5 (1996), 101-110. MR 97h:51015

9. _ An Atlas of residually weakly primitive geometries for small groups, Mém. Acad. Royale Belg., Classe des Sciences (1996), To appear.

10. , On flag-transitive incidence geometries of rank 6 for the Mathieu group $M_{12}$, Groups and Geometries (A. Pasini et al., eds.), Birkhäuser, 1998, pp. 39-54. CMP 99:01

11. F. Buekenhout and D. Leemans, On the list of finite primitive permutation groups of degree $\leq$ 50, J. Symbolic Comput. 22 (1996), 215-225. MR 97g:20004

12. - On a geometry of Ivanov and Shpectorov for the O'Nan sporadic simple group, J. Combin. Theory Ser. A 85 (1999), 148-164.

13. J. Cannon and W. Bosma, Handbook of Magma functions, Department of Pure Mathematics, University of Sydney, November 1994.

14. P. Cara, Truncations of inductively minimal geometries, Preprint, 1997.

15. P. Cara, S. Lehman and D. Pasechnik, On the number of inductively minimal geometries, Theoret. Comput. Sci. (to appear)

16. J.H. Conway, R.T. Curtis, S.P. Norton, R.A. Parker, and R.A. Wilson, Atlas of finite groups, Oxford U.P., 1985. MR 88g:20025

17. H.S.M. Coxeter and W.O.J. Moser, Generators and relations for discrete groups, second ed., Springer-Verlag, 1965. MR 30:4818

18. M. Dehon, Classifying geometries with Cayley, J. Symbolic Comput. 17 (1994), 259-276. MR 91f: 51007

19. M. Dehon and X. Miller, The residually weakly primitive and $(I P)_{2}$ geometries of $M_{11}$, In preparation. 
20. - The residually weakly primitive and $(I P)_{2}$ geometries of $U(4,2)$, In preparation.

21. M. Schönert et al., GAP Version 3.4, Lehrstuhl D für Mathematik, RWTH-Aachen, 1995.

22. H. Gottschalk, A classification of geometries associated with PSL(3,4), Diplomarbeit, Giessen, 1995.

23. H. Gottschalk and D. Leemans, The residually weakly primitive geometries of the Janko group $J_{1}$, Groups and Geometries (A. Pasini et al., ed.), Birkhäuser, 1998, pp. 65-79. CMP 99:01

24. D. Leemans, The residually weakly primitive geometries of the dihedral groups, Atti Sem. Mat Fis. Univ. Modena (to appear).

25. — The rank 2 geometries of the simple Suzuki groups $S z(q)$, Beiträge Algebra Geom. 39 (1998), no. 1, 97-120. CMP 98:10

26. — Thin geometries for the Suzuki simple group $S z(8)$, Proc. Third Int. Conf. on Finite Geometries and Combinatorics (F. De Clerck et al., ed.), vol. 5, Bull. Belg. Math. Soc. - Simon Stevin, 1998, pp. 373-387. CMP 98:14

27. _ The residually weakly primitive geometries of the Suzuki simple group Sz(8), Proceedings of Groups St Andrews 1997 in Bath (C.M. Campbell et al., ed.), CUP, To appear.

28. P. McMullen and E. Schulte, Regular polytopes in ordinary space, Discrete Comput. Geom. 17 (1997), 449-478. MR 98d:52014

29. J. Tits, Géométries polyédriques et groupes simples, Atti 2a Riunione Groupem. Math. Express. Lat. Firenze (1962), 66-88.

Université Libre de Bruxelles, Département de Mathématique, C.P.216- Géométrie, Boulevard Du Triomphe, B-1050 Bruxelles

E-mail address: dleemans@ulb.ac.be 